\title{
Research on Some Problems on Endurance Training Effectiveness Guang-bin BAl,a
}

\author{
Department of Physical Education, XIDIAN University , Xi'an 710071, China \\ abgb1972@126.com
}

Keywords: Endurance event; Training; Actual effect

\begin{abstract}
Adoption documentary summary and logical analysis, we discuss the problems and matters needs attention during specific endurance training The result shows that there is a common problem in specific training, that is the weakness in training effect Meanwhile, it leads to backward overall.. level of specific endurance training in China Conclusion and Advice:.. Combining with the interesting load and fast, effective effect further, emphasize on specific power endurance training Moreover, in order to improve training effect, we should enhance the aerobic exercise quality and aerobic- anaerobic based exercise quality further by economical limb action and physiological way. Training effect and concrete measure should be changed from qualitative analysis to quantitative analysis. According to individual condition, we'd better come up with a quantitative standard and particular training schemes.

Among the many international sporting events, the class of physical endurance sports projects, especially the lower level of the overall project, which is not only a low level of performance in track and field, swimming, rowing, cycling and other periodic road endurance events, but also in judo, wrestling and boxing and other non-cyclical sport, even in the process of training and competition football and basketball and other sports projects, due to the low specific endurance, strength endurance and speed endurance level and difficult to have a greater breakthrough. "Endurance" seems to have become sports quality individual athletes generally lacking. "Endurance" has become an obstacle to the low level of physical activity levels of projects to improve the outstanding issues of a universal, it can even lead to an imbalance of Sports Development, which restricts the full implementation of the Olympic gold medal strategy ${ }^{[1]}$. Endurance Events unsatisfactory results, to explore the reason can be described in many ways. The training effectiveness is not strong or endurance events has become a breakthrough and then medal bottleneck.

Essentially, the individual athlete inevitably limited by its own morphology, as well as environmental conditions. In order to achieve excellent results in competitive sports, athletes would have to obtain maximum development in the physiology, technical level and quality of mental aspects, so that the athletic ability in this particular limitation optimize ${ }^{[2]}$. Effectiveness of both the concentrated expression of this optimization is sports training exercise training scientific objectives pursued. A large number of theoretical research and practical studies have shown that as longdistance running, walking, cycling, canoeing, skiing, marathons and other endurance events in the course of scientific research, though already obtained Athletes'Physical state change part of the law, but the training and athletes functional status, and mental ability to adapt to the degree of fatigue and recovery of coaches and researchers still need a common analysis, diagnosis, evaluation, decision-making and control. The need for further understanding of the law of endurance events, the further implementation of the rationalization of the training process design and control, development of training effectiveness of the evaluation criteria, and use it as a reference standard.
\end{abstract}

\section{To further improve the special strength endurance, speed, strength endurance and speed endurance training.}

Training means clear classification and has become highly concentrated one of the basic characteristics of modern endurance training. Modern athletic sports for athletes training load and load game increasingly high demand, only Endurance athletes, special strength and speed strength 
endurance, speed endurance level requirements are also getting higher and higher, in order to adapt to the game, especially is a high-level endurance athletes training combined with a greater emphasis on specific projects, greater emphasis on specialized endurance training, general physical training the body as a mere means of recovery. On the training methods to change the traditional multimuscle strength endurance training methods, shift to local development in order to achieve a single muscle gradually improve special strength, strength endurance and speed endurance methods. State of the game features a variety of training methods and means and the load characteristics of the body closer to the athletes in the training process at each stage of the training mission revolves around the game of choice requires purposeful training means. Screening of endurance quality indicators project performance and endurance gray relational analysis results optimization program ${ }^{[3] 4]}$, the establishment of endurance athletes level indicator system, and then build endurance structural characteristics of elite athletes, sports training more targeted guidance.

\section{Aerobic and aerobic - anaerobic endurance training to improve the quality basis}

Basic supply system endurance events is aerobic energy supply system. Endurance in the foundation directly expressed as "aerobic capacity", "aerobic training" is an important part of endurance training. Ignored in the development of theoretical understanding and endurance training Training direction aerobic capacity, lack of effective training methods to improve aerobic capacity in endurance training practice, is responsible for the majority of Physical endurance sports projects, especially the main reason behind the long-term project one. However, the "endurance" training is a very complex issue, it is not only related to the nervous - muscular system, but also with the body's energy metabolism system has a very close relationship. Currently physiological body movement, there are still many aspects of biochemical mechanisms not yet conclusive or controversial issues, such as, "Fatigue mechanism", "after high-intensity muscle stimulation causes of pain" and so on, these problems are bound to affect " endurance "training. Had foreign researchers, endurance training on the autonomic nervous system regulation experimental research, endurance training can regulate the function of the autonomic nervous system has a good improvement ${ }^{[5]}$. Effects of different intensity endurance training on the autonomic nervous system has a significant difference. Effects of aerobic and anaerobic endurance training on the autonomic nervous regulation depends on the load strength training, and aerobic training is similar to the strict control of the appropriate intensity anaerobic endurance training may also help enhance the body's ability to adapt to the environment. This is for endurance athletes is critical. Load intensity of $80 \%$ to $90 \%$ of anaerobic endurance training to make the regulatory function of the autonomic nervous system or the enhanced role, thereby facilitating the body's adaptation to the environment ${ }^{[6]}$. For coaches and athletes, as much as possible about the project to master the Law of Development and Training Regularity. It has been shown to improve aerobic capacity of the training process, not simply an increase in training volume and intensity of training, aerobic need to understand the relationship between theory and training practice. From the perspective of sports physiology, sports biochemistry and Training of Athletes for different body strength and endurance training load, according to the characteristics of projects and selection of athletes training methods and means to develop detailed intensity grading, scientific testing and evaluation of aerobic capacity Effect of aerobic capacity and level of development of the special sports performance level that aerobic training and aerobic - anaerobic training content training, anaerobic training with different loads organically combine to form and characteristics of the project in line with the specific circumstances of athletes the best ratio, can effectively promote the development of both the level of endurance, but also can maximize avoid overtraining and sports injuries. Modern Sports events scheduled high frequency increases the proportion of special strength training athletes, Neumann swimming, cross-country skiing, marathon and triathlon world elite athletes long-term research, the results showed that the amount of accounting training Runners game $3 \%$ to $7 \%$ of the total, while the amount of annual road bicycle race world-class athletes of the total amount of training $35 \%$ to $40 \%{ }^{[7]}$. Therefore, high-intensity training needs throughout the entire training program of the whole story, but be sure to select training methods and means in accordance with sports features and 
characteristics of individual athletes to develop strength detailed classification to quantify standards guiding practical training.

\section{Economic limb movements}

3.1 Economic action forms the main component of acting and movement

Since Endurance is a typical relatively long duration exercise. So efficiency that can produce great benefits ${ }^{[8]}$. Bailey and Pate found little direct evidence that can improve the economic operation of elite distance runners, but they made it clear that pulmonary ventilation control, limb weight distribution, training status, body postures, which may be helpful to improve factors actions economy ${ }^{[9]}$. Stressing action at the same time pay attention to the economy and the economy of the action related to the case of mechanical power. Mechanical power means a person engaged in endurance activity rate of doing work. As can be seen from Table 1, the relative importance of some general endurance exercise done in several different types of work. Clear various forms of exercise do work main component and secondary component for improving the operation of the economy is necessary.

Table1 All kinds of sports acting ingredients

\begin{tabular}{lcccc}
\hline Category & $\begin{array}{c}\text { Overcome } \\
\text { gravity }\end{array}$ & Internal limbs & $\begin{array}{c}\text { overcome the fluid } \\
\text { resistance }\end{array}$ & Surface friction \\
\hline Running & $\mathrm{M}$ & $\mathrm{M}$ & $\mathrm{m}$ & - \\
bicycle & - & $\mathrm{m}$ & $\mathrm{M}$ & $\mathrm{m}$ \\
skiing & $\mathrm{m}$ & $\mathrm{M}$ & $\mathrm{m}$ & $\mathrm{M}$ \\
skating & - & $\mathrm{M}$ & $\mathrm{M}$ & $\mathrm{m}$ \\
boating & - & $\mathrm{m}$ & $\mathrm{M}$ & $\mathrm{m}$ \\
\hline
\end{tabular}

NOTE: M represents a main component; $\mathrm{m}$ represents a minor component; - represents a negligible component

3.2 Diastolic - contraction cycles and movements economy

Dastolic - systolic circulation system includes muscles, tendons and elastic tissue in the arch. It was generally believed that through relaxation - contraction cycle system can improve the operation of the economy, diastolic - systolic circulatory system to do mechanical work to reduce muscle movement in the metabolic process of the work done by the number ${ }^{[11][12][13]}$. Heise et al found an important link between the two electromyogram (EMG) indicators and running economy. They also noted a trend mentioned is to increase muscle activation time synchronizing can improve the economics of the operation. They assume that a large number of muscle fibers are activated simultaneously may cause joints stiffen, it would be involved in muscle movement and better recovery elastic potential energy, thereby improving the operation of the economy ${ }^{[14]}$. Other studies have found that the greater the elastic tissue while running the role, the more economic action ${ }^{[15]}$.

3.3 Training, flexibility and relaxation - contraction cycle system on economy

Diastolic - systolic circulatory system for acting role is unclear, but in the end was able to save energy metabolism by improving training. There is a reference to the view that flexibility training improves the ability to store elastic energy ${ }^{[16][17]}$. Some studies have tested the reduced physical flexibility for economic impact of the action. The study also found that, after a period of training flexibility, flexibility is improved, so that the economy is improved when running motion ${ }^{[18]}$. Morgan and Craib studies show that both are good or relatively good athletes, very good from an economic point of view the action, so action to optimize mechanical factors ${ }^{[19]}$.

People can quickly adapt to changes in motion, but the high level of the best action is needed after years of meticulous coordination to get. As more people understand the relationship between the mechanical action and the economy, stress in skeletal muscle and athletic performance and find the best application of this knowledge to all levels of athletes approach, biomechanical factors and sports-related fine regulation, athletes will become an important part of the training content. Therefore, in the implementation of the training process, focusing on the operation of economy requirements, exercise training is an effective means of improving the effectiveness. 


\section{Endurance and Endurance Performance psychological factors}

One problem endurance training Effectiveness implementation process can not be avoided is the intense psychological changes brought about by the pressure of endurance training have an adverse effect on some of the psychological factors as well as on the training effect on competition results, the study shows. Long-term physical activity or there is a significant correlation between sport and improve mental health ${ }^{[20]}$. But for the athletes, the effects of exercise on mental health is completely different, the training is also closely related to the pressure Athletes mood disorders degree. Whether training load is gradually increased in intensity physical activity or exercise, will increase the corresponding level athletes mood disorders ${ }^{[21]}$ (see Figure 1).

Psychological reactions to sportsmen and women engaged in similar endurance training arising from research, the results showed no significant difference ${ }^{[2]}$. Thus, during the course of endurance training, always pay attention to changes in physical activity and emotional changes athlete, try to prevent the pressure caused by the high intensity training significantly alter the emotional state of the athletes, to avoid emotional disorders, should become coaches and researchers noticed new problems.

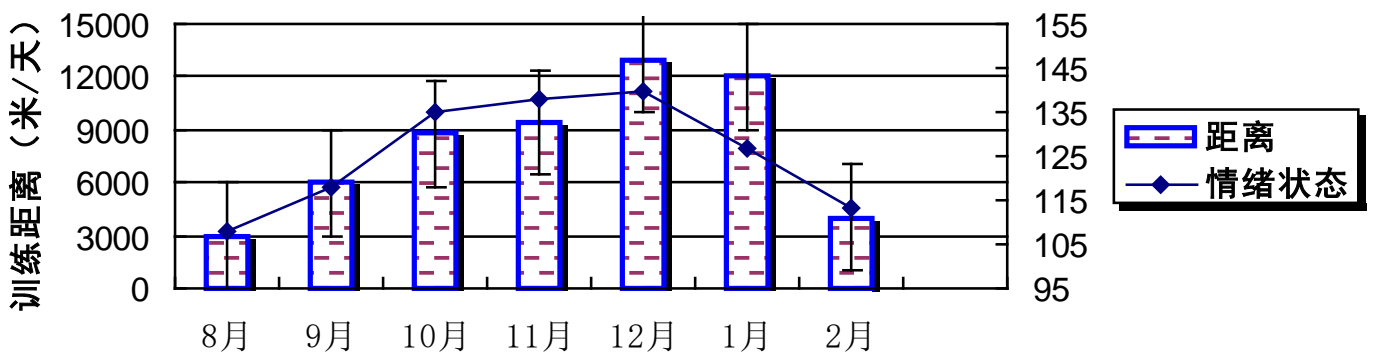

图1 训练量与情绪变化示意图

\subsection{Psychological methods of effective interventions and endurance sports performance}

Using imagery or imagery training training is endurance training effective psychological intervention. Image Training Act to improve athletic performance possible mechanism yet to be determined, a popular explanation is called the neuromuscular psychological theory. This theory assumes that, by the operation of the imagination, causing physiological changes activity corresponding to this action of the motor cortex and peripheral such as EMG (EMG), heart rate (HR) and the like. Experimental studies have shown that exercise can improve the appearance of the effect of endurance training.

4.2 Endurance exercise in cognitive factors - related to the association and toassociate

Morgan and Pollock reported that endurance athletes will always pay attention on such as pain, fatigue, muscle aches, breathing and body temperature such feeling, this is called cognitive strategies associated with it including the collection generated by body motion feedback, and use of these information adjust the exercise rhythm. In contrast, using a variety of ways to transfer physical exertion and discomfort of attention, this is called cognitive strategies related to ${ }^{\text {[23] }}$. Morgan noted that the use in the race to the associated methods, may make athletic performance below normal levels ${ }^{[24]}$. Morgan recently proposed that elite athletes associated with the use of cognitive strategies may be more procedural than non-elite athletes, but also to the associated policy is applied is not limited to the game. The fact that psychological factors have an impact on endurance exercise performance. Certain personality characteristics match their elite athletes to achieve good results in a variety of sports closely. 


\section{Rethinking load, fatigue and recovery means}

\subsection{Increase the training load on the basis of special ability}

High Intensity Training has become an important means of modern high-level training for endurance athletes. Endurance training load increase not only in quantity, better performance in strength. To increase the strength of the ways to improve the load, in order to induce the body to produce excess recovery better effect, a high level of total load either by highlighting the control load intensity reached, can also be achieved by projecting load control intensity ${ }^{[25]}$. Improve the level of training is based on the formation of a new body to adapt after stimulation, while the training intensity to stimulate the body's sensitivity to the most intense reaction. So, in terms of training load arrangements must be consistent with the strength of the body's tolerance to raise the highest value. In each period the training program, strength training should pay attention to this factor. Even in the preparation period and must meet greater strength speed endurance, strength and endurance training Endurance Training. Here it is worth to note that the increase in training load shall be carried out under the protection of sound restoration measures.Due to structural changes in the intensity of the load, the current domestic and international elite athletes in training before the game, the manifested an adjustment without special training, to take the edge training while adjusting training methods, so that the body is stimulated to recover as soon as possible a new trend. Further emphasized the effectiveness of training that emphasizes the close combination of increased training load and effective recovery of the body.

5.2 Closely integrated with the body increase the effective recovery of the training load

Matvei Fu also pointed out: exercise training is a complete, highly complex, multi-layered process ${ }^{[26]}$. Scholars have reached a consensus that exercise training as a systematic project, need to restore the body after training load closely linked rather than isolated treated differently, highlighting the characteristics of exercise training is scientific, practical, through sports training, the overall function of the athlete into a directional output having a relatively steady state or metastable said that the trained athlete who has become a special motor function ${ }^{[27]}$. Therefore, the scientific understanding of the basic characteristics of modern sports training and recovery, for improving the effectiveness of exercise training to improve athletic performance is important.

5.3 Re-examine the "Excess Recovery" Theory

"Excess Recovery" theory is widely used in sports training practice, and was regarded as coaches training and competition to improve the ability of magic. While this theory from the creation to the present has been the subject of some physiology and training experts questioned and challenged. The main question and challenge the lack of adequate scientific support for the theory of experimental data. "Excess Recovery" theory simply does not rely on experimental data under the strict control of experimental conditions obtained in vivo glycogen metabolism, with the result that the degree can not be easily extended to adapt to the different mechanisms of the entire body organs and systems will be explained. "Excess Recovery" theory did not exceed the limits of human ability, but in fact the body's ability of each system are influenced by heredity and other factors, their development is limited. "Excess Recovery" function increase theory emphasizes the short-term behavior, while ignoring the long-term cumulative effects. Based on the "excess recovery" theory or suspicion negative attitude in recent years, a number of German training expert industry training to adapt to the occurrence, development and change processes were studied. More typical of theoretical research is to adapt to Mader's "performance reserve model" [28] and Neumann "change - time to adapt dynamic" theory ${ }^{[29]}$.

"Excess Recovery" Although the theory has been questioned by some scholars, but for endurance athletes training in terms of excess recovery theory is still applicable. Within a certain range, the greater the amount of muscle activity, the degree of excess recovery appears more obvious, but the time delay occurred; on the contrary, the less energy consumed substances, the effect of excess recovery of the more obvious, but appeared earlier "in the over-recovery for the next stage of training, the best" that the previous recovery phase load of excess load is applied again to the body, the body will continue to improve the level of sports training practice has proved that athletes in 
excess recovery phase participate in training or competition, improve training effect, and will create an excellent competition results. Thus, according to coach the athletes own characteristics, excess recovery theory arrange training programs designed to make the training load athletes sustained and effective growth.

\subsection{Effective method to recover the body and means}

Endurance training effectiveness is not strong is a prominent feature of the training and recovery or can be said that the high intensity training and the "excess recovery" does not reach unity. An important feature of modern sports training, attention is back in training athletes, using a variety of means, measures to accelerate the recovery of the body. Modern training theory: "Recovery is trained to ensure that" "no recovery no training", "training is a continuation of the recovery," these views are profoundly reveals the importance of recovery after training ${ }^{[2]}{ }^{[30]}$.

In endurance athletes increasing emphasis on special strength endurance, speed endurance training situation, to resume training role has become increasingly important. In a sense, who resumed training one might do well to win the race. With the in-depth study of the recovery training, there are a variety of advanced recovery tools, methods in practice a wide range of applications, and with the establishment of full-time researchers athlete recovery center for athletes recover after a high-intensity training training provides good protection, and strive to create excellent athletes in sports performance, but also make full use of all possible conditions improve physical health. It is also training Effectiveness embodying an aspect. Currently, the top athletes at home and abroad to take advantage of all the conditions to accelerate the recovery process, in which the body motion fatigue recovery system mainly uses massage, stretching exercises, hydrotherapy, physiotherapy, oxygen and other means to accomplish; mainly used to restore the nervous system and internal environment absorbing negative oxygen ions, means qigong, music can enhance recovery and restoration take effect, speed recovery of a variety of drugs to complete, attention to psychological recovery of athletes, long-term high-intensity endurance training athletes likely to cause mental fatigue, so tired of training situation.

\section{Summary}

Effectiveness of exercise training as a modern sports training complex system in one of the most important constraint has been the majority of coaches, researchers are concerned. Effectiveness of endurance training performance is multifaceted, in the actual operation still need to pay attention to the training of many problems. Improve the quality of training is not by one or two high-quality action or one or two high-quality training courses will be able to decide, which is reflected in a long process of scientific and systematic training each and a small part on. Endurance Events behind the overall level of competitive sports is long-standing problems. To solve this problem, it will take a long time, we need to do a lot of basic research and practice of innovation. Only by constantly improving the quality of training to enhance awareness of the requirements and develop specific measures to be implemented consistently, you can really produce results. For endurance training law needs further study in order to have the appearance of scientific research update.

\section{Acknowledgements}

This work was financially supported "2014 Shaanxi Province Social Science Fund Project(2014N10)”

\section{References}

[1] Chen Xiaoping aerobic training - to improve our endurance sports level key projects [J] .2004 Physical Sciences, (24) 11: 45- 50.

[2] Rui Yuan exercise physiology [M].Beijing: People's Sports Publishing House, 2002. 
[3] Huang Ming belief, Chen Zhiqiang, Ding Ling and other sports Test design and scientific quantification methods [M]. Beijing: Higher Education Press, 2003.

[4] Xiaoxin Ping, Song Zhongmin, Li Feng. Ash technical basis and its application [M].Beijing: Science Press, 2005,136 142.

[5] James BC, Eric WB, Andrew PB Effect of endurance exercise on autonomic control of heart rate Sports Med, 2003,33 (1): 33.

[6] Open a new field, Qin Jun, Huang Lan Effects and significance of aerobic and anaerobic endurance training on the autonomic nervous regulation $[\mathrm{J}]$. Journal of Biomedical Engineering,2006; 23 (5): 1020 - 1023 .

[7] NEUMANN G, PRUTZNER A, BERBALK A. Optimiertes Ausdauer Training [M].Aachen: Meyer \& Meyer Verlag, 2001.

[8] Williams, K.R (1990). Relationships between distance running biomechanics and running economy In:Cavanagh, P.R (ed.) Biomechanics of Distance Running, PP.271-305.Human Kinetics, Champaign, IL.

[9] Bailey, S.P. \& Pate, R.R (1991) Feasibility of improving running economy. Sports Medicine 12, 228-236.

[10] R.J Shepard, P.-O Astor de Endurance [M].Beijing: People's Sports Publishing House, 2004,243-259.

[11] Williams, K.R. (1985) The relationship between mechanical and physiological energy estimates. Medicine and Science in Sports and Exercis17, 317-325.

[12] Taylor, C.R. (1994) Relating mechanics and energtics during exercise. Advances in Veterinary Science and Comparative Medicine 38A, 181-215.

[13] Alexander, R.M. (1991) Energy-saving mechanisms in walking and running. Journal of Experimental Biology160, 55-69.

[14] Heise, G.D, Morgan, D.W., Hough, H. \& Craib, M. (1996) Relationship between running economy and temporal EMG characteristics of bi-articular leg muscles. International Journal of Sports Medicine17, 128-133.

[15] Voigt, M, Bojsen-MILer, F., Simonsen, EB \& Dyhre-Poulsen, P (1995) The influence of tendon Youngs modulus, dimensions and instantaneous moment arms on the efficiency of human movement.Journal of Biomechanics 28, 281-291.

[16] Wilson, G.J, Wood, G.A. \& Elliott, B.C. (1991) Optimal stiffness of series elastic component in stretch-shorten cycle activity. Journal of Applied Physiology70, 825-833.

[17] Wilson, G.J., Elliott.B.C. \& Wood, G.A. (1992) Stretch shorten cycle performance enhancement through flexibility training. Medicine and Science in Sports and Exercise24, 116-123.

[18] Godges, JJ, Macrae, H., Londgon, C. \& Tinberg, C. (1989) The effects of two stretching procedures on hip range of motion and gait economy. Journal of Orth opaedic and Sports Physical Therapy7, 350-357 .

[19] Morgan, D.W. \& Craib, M. (1992) Physiological aspects of running economy.Medicine and Science in Sports and Exercise24,456-461.

[20] Morgan, W.P. (1997b) Physical Activity and Mental Health.Taylor \& Rrancis, Washington, DC.

[21] Raglin, J.S., Eksten, F. \& Garl.F.T. (1995) Mood state responses to a pre-season conditioning program in male collegiate basketball players. International Journal of Sport Psychology26, 214225. 
[22] O'Connor, P.J., Morgan, W.P. \& Raglin, J.S. (1991) Psychobiologic effects of 3d of increased training in female and male swimmers. Medicine and Science in Sports and Exercise23, 1055-1061.

[23] Morgan, W.P. \& Pollock, M.L (1977) Psychologic characterization of the elite distance runner. Annals of the New York Academy of Science301, 382-403.

[24] Morgan, WP (1997a) Mind games: the psychology of sport In: Lamb, DR \& Muray, R. (eds) Optimizing Sport Peformance: Perspectives in Exercise Science and Sports Medicine, Vol.10, pp.154 .Cooper Publications, Carmel, IN.

[25] Xu Benli adaptation, load and recovery theory [J].Shandong Institute of Physical Education Daily, 2001, 17: (4).

[26] Column Matvei EDUCTION husband also translated the 11th Asian Games theme of the report is selected,To arrange sports training principles and laws [J] .Sport Science, 1991.6.

[27] Han Dan. On the sports training system structure and function optimization [J]. Fujian Sports Science, 1991,41- 46.

[28] Mader A. A ktive Belastungsadaptation and Regulation der Proteinsynthese auf zellulaerer Ebene [J].Deutsche Zeitsch rift Sport medizin, 1990; 41: 40 - 58.

[29] Neumann G. Schuele K.-P. Sport medizinische Funktions-diagnostik [M].L eipzig Berlin Heidelberg: Barth V erlag-sgesellschaft, 1994: 13 - 17.

[30] Tian Maijiu Sports Training [M]. Beijing: People's Sports Publishing House, 2000. 